\title{
Health Evaluation Model of Important Equipments in Intranet Based on AHP and Entropy Analysis
}

\author{
Xinmei Hou ${ }^{a,}{ }^{*}$, Kaikun Dong ${ }^{b}$ \\ School of Harbin Institute of Technology University, Harbin 150001, China. \\ a610636654@qq.com, b1315989193@qq.com
}

Keywords: correlation coefficient; entropy analysis; analytic hierarchy process; health evaluation.

\begin{abstract}
This paper constructs a general equipment health evaluation model for the operation state of important equipments in intranet, which evaluates the health of equipments from three aspects: key performance index, key quality index and quality of experience index. The weight of important state indexes are calculated with the combination of analytic hierarchy process and entropy analysis. In addition, this paper use correlation coefficient to revise the weight deviation caused by the association among performance, professional ability, and user experience. The health of running status of servers under different pressure gradients were evaluated, the experimental results showed that the corrected health evaluation values under different pressure states were reasonable and satisfactory, which was in accordance with actual application scenarios. In addition, the degree of health correction has a significant effect when the health of the server is less than 60. Therefore, the evaluation system can provide reliable basis for enterprises to monitor the running status of important equipments in intranet.
\end{abstract}

\section{Introduction}

Recently, computer technology has penetrated into all walks of life, the operation security problems of enterprise intranet are ensued. Enterprise intranet resources contain a large number of network equipments and application servers. Taking the servers as an example, in [1] Yifan Wang et al, introduced the concept of "software decay" and analyzed the rules of system performance degradation by AHP algorithm. In [2, 3] authors considered system performance to build the evaluation model. In [4] the author took the server service capability as the evaluation index including the average response time, throughput and concurrency. This paper constructs a general health evaluation model of important equipments of Intranet, and takes web application server as an example to illustrate the construction process of model. In this paper, the operating status data of the servers under different pressure conditions were collected from the three aspects of KPI (Key Performance Indication), KQI (Key Quality Indicator) and QoE (Quality of Experience). After selecting the important indicators and calculating the entropy weight scores, the health of the server by analytic hierarchy process could be calculated, and the Spearman's rank correlation coefficient to correct the deviation of KPI, KQI and QoE could be calculated, and at last the final health score could be got to reflect the service capability of server.

\section{Related Theoretical Basis and Methods}

\subsection{Entropy Right Method}

An objective scoring criteria based on entropy weight in the grading process of AHP was provided. The formula for calculating the entropy is shown in Equation 1.

$e_{j}=-k \sum_{i=1}^{n} p_{i j} \ln p_{i j}$

In equation $1,1 / \ln (n)>0, e_{j} \geq 0, p_{i j}$ represents the contribution of the sample $\mathrm{i}$ to the index $\mathrm{j}$.

The formula for calculating the weight of the indexes is shown in Equation 2. In equation $2, d_{j}$ represents the utility value of index $\mathrm{j}$.

$$
w_{j}=\frac{d_{j}}{\sum_{j=1}^{m} d_{j}}, d_{j}=1-e_{j}
$$




\subsection{Analytic Hierarchy Process}

The key of the AHP is to construct a pairwise comparison matrix that could meet consistency check. The consistency coefficient $\mathrm{CR}$ is used to determine whether the matrix passes the consistency check. If CR $<0.1$, it can be considered that the matrix has satisfactory consistency.

\subsection{Correlation Coefficient Calculation}

The fine-grained indexes of KPI, KQI and QoE were mutual influenced. Therefore, in this paper, the related calculation between correlation coefficients was used to correct the weight deviation. The Kolmogorov-Smirnov test was used to test the data samples, and the Spearman coefficient was selected to reflect the association between the fine-grained indexes.

\section{Health Evaluation Model}

\subsection{Index}

KPI (Key Performance Index) is measurement of the performance quality of a single or a group of business resources. In this paper, the performance test tool named "nmon" was used to passively collect KPI performance parameters of web application server. The selected KPI indexes were collected including mem, cpu, diskbusy, net and blocked.

KQI (Key Quality Index) describes the service quality directing at different applications provided by servers. In this paper, the test tool named "loading" was used to actively collect the KQI indexes, the collected indexes were collected including 'fetches/sec(f_s)', 'bytes/sec(b_s)', 'bytes /connect (b_c)', 'msecs/connect(m_c)' and 'msesc/first-response(m_f)'.

QoE (Quality of Experience) directly reflects the user's subjective experience on the service of application. In this paper, the QoE indexes of web application servers were actively collected to determine the indexes that directly related to UE. The QoE indexes were collected including 'resolve', 'connect', 'request', 'process' and 'close'.

This chapter mainly describes the key indexes of the evaluation model and the process of selection. Besides, the calculation methods of KPI, KQI and QoE fine-grained indexes are elaborated.

\subsection{Analytic Hierarchy Model}

The entropy weight of KPI, KQI, QoE fine-grained indexes were calculated with formula 1 and 2, then it was used to mark the fine-grained indexes. The scoring rules of fine-grained indexes are based on the entropy weight which calculated with equation 3. The rules are shown in Table 1.

\begin{tabular}{|c|c|c|}
\hline & order & description \\
\hline 1 & KPI & mem $>$ net $>$ cpu $>$ diskbusy $>$ blocked \\
\hline 2 & KQI & $\mathrm{m}_{-} \mathrm{f}>\mathrm{m}_{-} \mathrm{c}>\mathrm{f} \_\mathrm{s}>$ fetches $>\mathrm{b} \mathrm{c}>\mathrm{b} \_\mathrm{s}$ \\
\hline 3 & QoE & write $>$ request $>$ com $>$ resolve $>$ close \\
\hline
\end{tabular}

The hierarchical structure model of health evaluation is shown in Figure 1 . Based on the scoring rules and this model, the relevant pairwise comparison matrices were constructed.

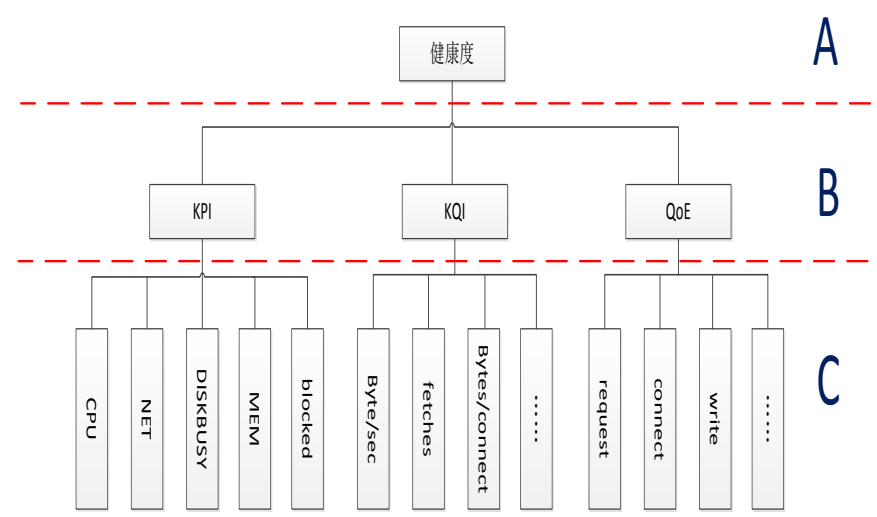

Figure 1. Hierarchical Structure Model of Health Evaluation

\subsection{Fine-grained Index Correlation Analysis}

The weight correction coefficients between KPIs, KQI and QoE are calculated by the correlation coefficients between fine-grained indexes. The calculation method is shown in Equation 3. $\mathrm{m}$ 
represents three upper indexes including KPI, KQI and QoE, $r_{i j}$ represents the correlation coefficient between fine-grained index $\mathrm{i}$ and the fine-grained index $\mathrm{j}$.

$$
c_{j}=\left(\sum_{j}^{m} 1-r_{i j}\right) / \sum_{i} c_{i}, \mathrm{i} \in[1,3], \mathrm{i} \in Z^{*}, r_{i i}=1
$$

\subsection{Health Evaluation Model}

The model uses the fine-grained indexes of KPI, KQI and QoE as input, and the server health score is used as output to construct the health evaluation model. The calculation of server health is shown in Equation 4. $\mathrm{i}=1,2,3$ represents the index of KPI, KQI and QoE; $m$ is the number of fine-grained indexes of upper index $i, C_{i}$ is the weight correction coefficient of $i, W_{A i}$ is the weight coefficient of $\mathrm{i}, \mathrm{w}_{\mathrm{j}}$ is the fine-grained index $\mathrm{j}$ of $\mathrm{i}, \mathrm{A}_{\mathrm{j}}$ is the running state of the current fine-grained index $\mathrm{j}$. According to the equation 4 , the reliable health of server could be obtained.

$$
\mathrm{Q}=\sum_{i=1} \sum_{j=1}^{m} w_{j} A_{j} C_{j} W_{A_{i}}
$$

\section{Results and Analysis}

In this paper, a web application server was built to measure the health of server under 50 different gradients. The results of weight calculated with and equation 4 are shown in Table 2. It suggests that the fine-grained indexes, including memory, the average response time of the page, the time of the handling request by server et al, have great significance on the health of server.

\begin{tabular}{|c|c|}
\hline weight & description \\
\hline $\mathrm{W}=\left[\begin{array}{llllll}0.4785 & 0.0751 & 0.2800 & 0.1189 & 0.0475\end{array}\right]^{\mathrm{T}}$ & $\mathrm{w}=[\text { mem diskbusy net cpu blocked }]^{\mathrm{T}}$ \\
\hline $\mathrm{W}=\left[\begin{array}{llllll}0.4340 & 0.2040 & 0.1168 & 0.1347 & 0.0721 & 0.0358\end{array}\right]^{\mathrm{T}}$ & $\mathrm{W}=\left[\mathrm{m} \_\mathrm{f} \mathrm{m} \_\mathrm{c} f \_\mathrm{s} \text { fetches b_c b_s }\right]^{\mathrm{T}}$ \\
\hline $\mathrm{w}=\left[\begin{array}{lllll}0.2506 & 0.1334 & 0.5048 & 0.0665 & 0.0447\end{array}\right]^{\mathrm{T}}$ & $\mathrm{w}=[$ request connect write $\overline{\text { resolve }}$ \\
\hline close $]^{\mathrm{T}}$ & \\
\hline $\mathrm{W}=\left[\begin{array}{llll}0.5396 & 0.2970 & 0.1634\end{array}\right]^{\mathrm{T}}$ & $\mathrm{W}=[\mathrm{KPI} K Q I \mathrm{QoE}]^{\mathrm{T}}$ \\
\hline
\end{tabular}

Table 2. Weight

The correlation coefficients between KPI, KQI and QoE fine-grained indexes were calculated. The correction coefficients are shown in Table 6.

Table 3. Weight Correction Coefficients

\begin{tabular}{clll}
\hline index & KPI & KQI & QoE \\
\hline coefficient & 4.0942 & 2.9406 & 2.5381 \\
\hline
\end{tabular}

The difference between the corrected and the uncorrected server health at 50 pressure gradients is shown in Figure 2. The orange column represents the uncorrected health score, and the black column represents the corrected health score. In this paper, the related calculation of correlation coefficient was used to modify the deviation. Figure 3 shows the correction degree of deviation. The red column represents the health deviation tendency before and after correction. The black column represents the deviation of the correction under different pressure conditions.

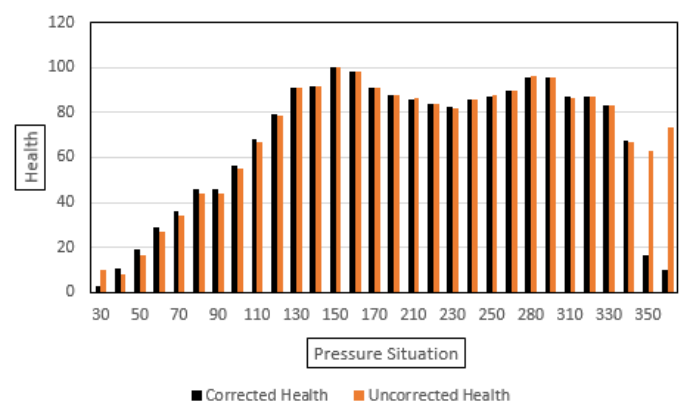

Fig 3. Comparison of Health of Server

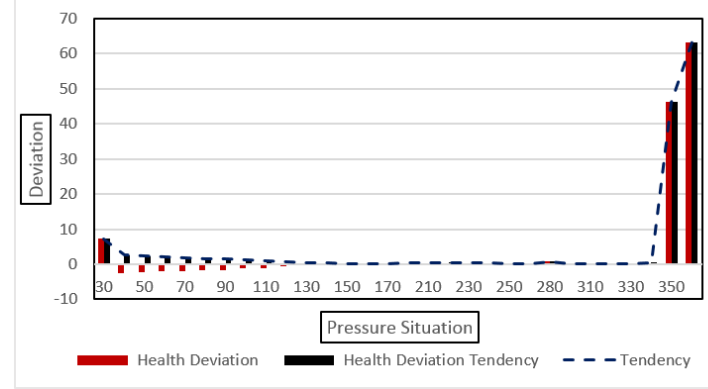

Fig 4. Degree of Server Health Deviation

It could be seen from Figure 2 and 3 that when the server concurrent pressure is less than 100, the server health is basically less than 60. As a result, the correlation between KPI, KQI and QoE finegrained indexes will have a certain impact on health evaluation. This phenomenon reflects the waste of resources in intranet. Meanwhile, the network topology architecture should be adjusted. When the server concurrency is greater than 300, the health of the server decreased dramatically, and the deviation degree of health greatly increased. It means that the server almost has no service capacity 
when being busy to a certain extent. At the meantime, maintenance personnel should take appropriate measures to maintain. When the server concurrency is between 100 and 300, the server has reasonable usage of business and resources, as well as satisfactory health.

It demonstrates that the correlation between the KPI, KQI and QoE fine-grained indexes has a significant impact on health.

\section{Summary}

In this paper, a general health evaluation model was proposed from the perspective of the running status of the enterprise intranet. The health of the equipments was evaluated from the aspects of KPI, KQI and QoE by using the analytic hierarchy process, and the entropy weight method provided objective scoring basis for analytic hierarchy process. Through the correlation analysis of the KPI, KQI and QoE fine-grained indexes, it could be found that there was a significant correlation, the correction coefficient was used to correct the weight deviation caused by the association in the analytic hierarchy process. After testing, it demonstrated that the model could make a reasonable evaluation of the operation status of the equipments, and provide reliable basis for the operation monitoring of the equipments in enterprise intranet.

\section{References}

[1] Yifan Wang, Guixi Xiong. Research for a System Performance Degradation Detection Method based on AHP [J].Journal of Microcomputer Information, 2010,(30):159-161.

[2] Haining Meng, Yong Qi, Di Hou. Modeling and analysis of application server reju venation based on periodical system performance inspection [J]. Journal of NanJing University, 2010, 46(4):440-447.

[3] Chuanyi Liu, DongshengWang. AHPCC: A High Performance Computer System Evaluation Model Based on HPCC and Analytic Hierarchy Process [J].Journal of Software, 2007, 18(4): 1039-1046.

[4] Zheng Zhang, Hongpeng Wang, Jingtai Liu. Performance Analysis and Optimization of Network Server Based on Node Cache [J]. Computer Engineering, 2012, 38(1):5-9. 